\title{
Membership function model for defining optimality of vapor pressure deficit in closed- field cultivation of tomato
}

\begin{abstract}
Estimation of plant's evapotranspiration (ET) or water loss to the atmosphere depends on the vapor pressure deficit (VPD) of the closed-field environment (greenhouse). The objective of this work was to develop a membership function model for defining optimal VPD of greenhouse air for tomato cultivation (Lycopersicon esculentum) at different growth stages (GS) and light conditions (sun, cloud, night). Mathematical descriptions of a peer-reviewed published growth response (GR) model for optimal greenhouse air temperature $(\mathrm{T})$ and relative humidity $(\mathrm{rH})$ were derived and implemented in a computer program. An incremental algorithm was written in MATLABC based on definitive concepts in VPD equations and the GR model. Non-linear regression was applied to describe mathematical relationship between the incremented outputs of the model and the calculated VPD values (R2=0.999 to 1 ). Results were validated with three published literatures and were shown to be capable of exploring optimal levels of VPD by means of real numbers between 0 and 1 . This study can contribute to knowledge-based information and decision support systems in greenhouse climate control and management by quantifying comfort level of microclimate.
\end{abstract}

Keyword: Vapor pressure deficit; Optimal value; Greenhouse; Tomato; Growth response; Membership functions 